\title{
Arbeitskreis Kinderurologie: Frankfurt, Januar 1993 Thema: Rhabdomyosarkome
}

\author{
D. Sternberg \\ Urologische Universitätsklinik Mainz
}

Die Einleitung zum Thema Rhabdomyosarkome erfolgte in Form eines "State of the Art"-Vortrags durch P. Gutjahr (Mainz). Unter den malignen Weichteiltumoren im Kindesalter sind mit $65 \%$ Rhabdomyosarkome die häufigsten. Diese sind embryonale Tumoren und lassen sich aus quergestreiften Muskelzellen ableiten, genauer aus undifferenziertem mesenchymalen Gewebe. Trotz ihrer ausgeprägten Tendenz zu Lokalrezidiven und hämatogener Metastasierung sind Rhabdomyosarkome (RMS) heute überwiegend heilbar und können in nahezu allen Geweben vorkommen.

Therapie und Prognose hängen zum großen Teil von Lokalisation und Ausdehnung der Erkrankung ab, therapeutisch sind Operation, Radio- und Chemotherapie, auch abhängig vom Stadium, in geeigneter Weise zu kombinieren.

Die häufigste Lokalisation des RMS ist in der Orbita, ferner auch im HNO-Bereich, den unteren Extremitäten sowie den Gallenwegen.

Die Ätiologie ist letztlich unklar, eine vermehrte Häufigkeit wurde familiär oder bei Patienten mit Neurofibromatose von Recklinghausen beobachtet. Das Durchschnittsalter bei Erkrankung liegt ungefähr bei 6 Jahren, jedoch mit 2 Altersgipfeln (3.-5. und 13.-18. Lebensjahr). Knaben werden häufiger betroffen als Mädchen, insbesondere bei Lokalisation des Urogenitaltraktes, wobei hier die Prostata und der Blasenhals häufiger als die übrige Harnblase dominieren (botryoides RMS). Bei Knaben können die Tumoren ferner primär von paratestikulären Weichteilen, Samenstrang, Prostata oder Skrotum, bei Mädchen von Ovar, Uterus und Vagina ihren Ursprung nehmen.

Zur Pathologie erwähnenswert, da $\beta$ bei makroskopisch unspezifischem Bild, mikroskopisch ein indifferentes mesenchymales Blastem herrscht mit verschiedenartigen, z.T. charakteristisch geformten Zellen (z.B. sogenannte Kaulquappen- oder Tennisschlägerzellen), die hyperchromatisch und insgesamt polymorph sind und quergestreifte Zellelemente nachweisen. Bei immunhistochemischen Färbungen sind diese meist Desmin-positiv. RMS metastasieren über den Lymph- oder Blutweg aus.

Akt. Urol. 24 (1993) 300-303

(C) Georg Thieme Verlag Stuttgart · New York
Die Klinik richtet sich nach Sitz und Ausdehnung der Tumoren und ist daher sehr variabel. Im Retroperitonealraum verursachen sie Symptome erst, wenn sie relativ groß sind: Unwohlsein, Obstipation, Diarrhö, „rezidivierende Harnwegsinfekte“ und Schmerzen sind die häufigeren unspezifischen Erstsymptome, aber auch hinter einer akut aufgetretenen Hämaturie, Dysurie oder Harnverhaltung kann sich ein RMS verbergen. Sie können als akutes Abdomen imponieren oder tasten sich zufällig als Resistenz. Bei Knaben kann die einseitige Hodenschwellung, bei Mädchen ein blutiger Ausfluß oder scheinbarer Vaginalpolyp Ausdruck eines RMS sein. Bei Lokalisation anderer Organe, wie Orbita oder HNO-Bereich, kann die rasche einseitige indolente Schwellung begleitende Symptome Hinweis für die Erkrankung bieten.

Nur die histologische Diagnosestellung bietet ausreichend Sicherheit; die klinischen, röntgenologischen (einschließlich CT sowie MRT) und sonographischen Befunde erlauben es kaum, die Artdiagnose mit absoluter Sicherheit vorauszusagen. Spezielle Tumormarker gibt es für die Rhabdomyosarkome nicht, ebenso keine serologische oder blutchemische Labortests.

Stadieneinteilung: Die Prognose des RMS ist wesentlich vom Stadium der Erkrankung, von der Therapie und vom histologischen Subtyp abhängig. Im Stadium I ist die Erkrankung lokalisiert, eine komplette Resektion kann durchgeführt werden, die regionalen Lymphknoten sind frei. Bei Stadium II erfolgte makroskopisch eine radikale Tumorentfernung, jedoch Nachweis von mikroskopischem Residualtumor mit oder ohne regionaler Lymphknotenbeteiligung. Stadium III bedeutet eine inkomplette Entfernung mit makroskopischem Residualtumor, wogegen in Stadium IV Fernmetastasen bei Diagnosestellung vorliegen.

Bei der Therapie im Kindesalter besteht kein Zweifel mehr an der erheblichen Bedeutung der Chemotherapie, dazu der Operation und Radiatio, diese einzelnen Verfahren haben jedoch einen unterschiedlichen Stellenwert je nach Stadium und Lokalisation des RMS. Wenn die Tumoren primär radikal operabel erscheinen, ohne größere Einbuße von Funktion und Kosmetik, steht das chirurgische Vorgehen an erster Stelle, insbesondere bei einem Großteil der Stadium I-RMS. Eine radikale Operation ist oftmals auch erreichbar, wenn eine präoperative zytoreduktive Chemotherapie angewendet wurde. RMS sind grundsätzlich radiosensibel, daraus ergibt sich, daß sowohl die präoperative als auch postoperative Radiotherapie zur Anwendung kommen kann. Wenn bei einem 
kindlichen RMS durch Operation der Primärtumor radikal entfernt werden konnte, so kann die spätere Bestrahlung entfallen, eine kurzfristige zytostatische Behandlung ist in jedem Fall angezeigt. In der Regel werden bezüglich der Bestrahlung 30-40-50 Gy angestrebt. Die Chemotherapie beinhaltet auch heute noch als Grundgerüst das sogenannte VAC-Schema (Vincristin, Actinomycin D, Cyclophosphamid). Beim VACA-Schema wurde noch Adriamycin hinzugefügt, als Modifikation wird beim VAIA-Schema das Cyclophosphamid durch Ifosfamid ersetzt. Letztlich fand auch Etoposid Eingang in die Therapie der Weichteilsarkome, insbesondere bei Hochrisiko-Patienten. Alternative Substanzen sind Cis-Platin, DTIC und Bleomycin. Im allgemeinen werden heute zytostatische Behandlungen in Zyklen von z. B. 10wöchiger Dauer durchgeführt und mehrfach wiederholt, ca. dreimal, was einschließlich der Erholungsphasen und einer evtl. Strahlentherapie zu einer Gesamtbehandlungsdauer von etwa 40 Wochen führt, natürlich in Abhänigkeit vom Stadium des Tumorgeschehens.

Verlauf und Prognose: Rhabdomyosarkome sind rasch proliferierende Geschwülste, sie neigen $\mathrm{zu}$ Lokalrezidiven, Lymphknoten- und hämatogenen Metastasen. Sie sind durch die moderne Behandlung in mehr als der Hälfte der Fälle mit primär nicht-metastastischen Erkrankungen dauerhaft beherrschbar geworden. Die Prognose ist naturgemä $\beta$ abhängig vom Stadium der Erkrankung, wobei für die lokalisationsunabhängige Beurteilung eine Prognose von mehr als $80 \%$ Dauerheilungen nach 3 Jahren für Stadium I gilt, für Stadium II eine solche von $70 \%$, jedoch in Stadium III und IV diese auf 20-50\% abfällt. Besonders große Tumoren haben eine deutlich schlechtere Prognose als kleinere, aber auch bei diesen ist, wie bei allen anderen, das initiale Ansprechen auf eine Chemotherapie ein kritischer prognostischer Faktor. Pauschal ergibt sich nach initialer Chemotherapie mit kompletter Remission eine Überlebenswahrscheinlichkeit von $89 \%$ nach 5 Jahren, im Vergleich dazu eine solche von lediglich $29 \%$ nach 5 Jahren, wenn der Tumor nur zu einem Drittel reduziert werden konnte. Rezidive und Fernmetastasen treten meist innerhalb der ersten beiden Jahren nach Erstdiagnose auf, nach mehr als 3 Jahren kann mit größter Wahrscheinlichkeit von einer permanenten Heilung die Rede sein. Die Prognose wird besonders ungünstig nach Rezidiv und Eintreten einer Metastasierung unter Therapie.

Es folgte danach der erste Beitrag über „die operative Strategie bei Rhabdomyosarkomen des "Urogenitaltraktes" von M. Fisch (Mainz). Da eine lokale Exzision oder partielle Zystektomie, wie sie von Hays et al. befürwortet wird, sehr risikoreich ist, sollte die Radikaloperation neben der präoperativen Chemotherapie Teil des Therapiekonzeptes sein. Multiple intraoperative zentripetale Biopsien mit Schnellschnittuntersuchungen, d.h. den Tumor eingrenzend, sind notwendig, da makroskopisch, insbesondere nach Chemotherapie, eine Differenzierung von vitalem und durch die Therapie devitalisiertem Tumorgewebe nicht mehr möglich ist. Es resultiert daraus eine ausgedehnte Lymphadenektomie. Als Harnableitung sollte eine kontinente Methode der Wahl sein, bei inkontinenter Harnableitung sei das Kolon-Conduit erwähnt, welches sich ideal für die antirefluxive Ureterim- plantation eignet, insbesondere unter dem Aspekt der möglichen Inkorporation des Conduits bei einer späteren Konversion in eine kontinente Harnableitung. Es wurde darauf verwiesen, daß bei geplanter Bestrahlung die Ureteren und die Harnableitung nach Möglichkeit außerhalb des Strahlenfeldes liegen sollten. Im Rahmen der anschließenden Diskussion wurde nochmals auf die Notwendigkeit einer radikalen Operation eingegangen.

H. G. Dietz (München) ging auf mehrere Studienkonzepte bei „Urogenitalen Rhabdomyosarkomen" ein, dabei wurden zwischen 1976 bis 1992 insgesamt 68 Kinder mit Rhabdomyosarkomen behandelt, darunter fanden sich 23 urogenitale RMS. Erst seit 1981 lagen entsprechende Protokolle vor. Nach dem CWS '81Konzept wurde nur ein Patient mit einem Rhabdomyosarkom der Blase behandelt, nach Diagnosestellung durch PE erfolgten die Bestrahlung, Chemotherapie und letztlich die Second-look-Op., wobei der Patient z.Z. in Vollremission überlebend ist. Im Rahmen des CWS '86 wurden $10 \mathrm{~Pa}$ tienten therapiert, in das jetzt gültige CWS ' 91 wurde bislang nur ein Patient mit paratestikulärem RMS aufgenommen. Von den 23 Patienten waren 16 männlich und 7 weiblich, mit einem Durchschnittsalter von 5,9 Jahren (0,7-15 Jahre) mit einer Lokalisation von Blase und Prostata $(n=12)$, Becken(-boden) $n=5$ und im sonstigen Urogenitaltrakt $(n=6)$. Die Raten des ereignisfreien Überlebens lagen zwischen 0,4 und 0,83 , wobei die Gruppen PE und Chemotherapie deutlich im Nachteil lagen. Ein Vergleich der internationalen Studien für das Rhabdomyosarkom im Urogenitalbereich zeigt für die SIOUPStudie 54\%, INTERGROUP 64\%, CWS ' 81 ca. $62 \%$ und die italienische Studie $78 \%$ Überlebende.

Ein Fallbericht zur „Blasenerhaltenden Therapie bei Rhabdomyosarkom der Prostata unter Einsatz der intraoperativen Radiatio" wurde von F. Kempter (Heidelberg) vorgestellt. Bei einem 3jährigen Knaben mit einem stanzbioptisch gesicherten Rhabdomyosarkom ohne Nachweis jeglicher Metastasierung - wurde nach einem Zyklus EVAIA ohne Ansprechen und nach weiteren 2 Zyklen mit modifiziertem Schema, wobei jetzt Carboplatin und Dacarbazin verwendet wurde, die chirurgische Therapie mit intraoperativer Radiotherapie (IORT) angewandt. Die operative Therapie bestand in der radikalen Prostatektomie. Die Harnblase mit negativen Absetzungsrändern wurde erhalten, die Lymphknoten und der Urethraabsetzungsrand waren ebenfalls tumorfrei. Da der Tumor aber an der Rektumvorderwand adhärent war, wurde die IORT angewandt, und zwar mit einem Linearbeschleuniger, wobei eine alleinige Elektronenbestrahlung erzeugt wurde, welche mittels einer speziellen Apparatur in das OP-Feld zur Applikation einer räumlich scharf begrenzten und homogenen Dosisverteilung geleitet wurde. In der anschließenden Diskussion wurde nochmals auf die möglichen Spätschäden verwiesen, zusammenfassend wurde der eher zurückhaltende Einsatz der Radiatio empfohlen.

V. Abramovic (Zagreb) berichtete über die „klinischen Erfahrungen mit Rhabdomyosarkomen der genito-urinären Organe bei Kindern“. 1988-1992 wurden 15 Kinder mit RMS verschiedener Lokalisationen behandelt, 5 davon im urogenitalen Bereich. Berücksichtigt 
wurden dabei die chirurgische als auch die übrige übliche Therapie im Rahmen der SIOUP-Protokolle. Die Ergebnisse ließen sich mit denen der bekannten Literatur vergleichen.

Es folgten die „Langzeitergebnisse bei paratestikulären Rhabdomyosarkomen" von J. D. M. de Vries (Nijmegen), wobei gezeigt wurde, daß die intraskrotale paratestikuläre Lokalisation etwa $7-10 \%$ aller RMS ausmacht. Es wurden 10 Patienten gemäß einem einheitlichen Protokoll zwischen 1979-1992 behandelt. Das Alter bei Diagnosestellung betrug 1,5-22 Jahre, 7 Patienten befanden sich im Stadium I, während die restlichen 3 dem Stadium III zugeordnet wurden. Von den Stadium-I-Patienten wurden bei 3 keine RPLA durchgeführt, weil keine lokale Invasion vorlag, bei 2 Patienten zeigte die RPLA negative Lymphknoten, bei den anderen 3 waren die Lymphknoten positiv (patholog. Stadium II). Alle Patienten im Stadium I erhielten eine zyklische Chemotherapie mit Vincristin und Actinomycin-D mit guten Ergebnissen bei abnehmender Zyklenzahl, bei den 2 Patienten im pathologischem Stadium II schloß sich an die RPLA eine Chemotherapie an, welcher Cyclophosphamid hinzugefügt wurde. Die 3 Patienten im Stadium III erhielten eine Chemotherapie für 4-6 Monate vor der RPLA. Bei einem Patienten mit Residualtumor folgte eine Radiotherapie mit $30 \mathrm{~Gy}$, danach eine Chemotherapie für 20 Monate, bei einem anderen mit Lungenmetastasen nach RPLA eine aggressivere Chemotherapie. Die Langzeitergebnisse zeigten ein tumorfreies Überleben bei 9 Patienten, wobei das mittlere follow up 84 Monate betrug. Hieraus ergab sich eine gute Prognose der paratestikulären RMS bei Anwendung einer kombinierten Therapie.

Zum Thema „Therapeutische Aspekte beim paratestikulärem RMS im Kindesalter" wurde anhand einer Kasuistik die Problematik durch E. Gottschalk (Erfurt) unterstrichen. Es wurde der Fall eines 14jährigen Knabens dargestellt, welcher wegen Lungeninfiltrationen auffiel bei ausgeprägtem Bild eines Hodentumors mit Infiltration des Retroperitonealraumes. Nach durchgeführter Semikastratio links folgte eine zytostatische Therapie konform der GPOH-CWS 91. Es zeigte sich eine gute Remission - einschließlich der pulmonalen Absiedlungen. Nach erfolgter RPLA des Resttumors ist eine Ganzkörperbestrahlung zur Remissionssicherung geplant.

R. Romanowski (Essen) stellte die „regionale Hyperthermie und systemische Chemotherapie bei Kindern mit lokalisierten Hochrisikotumoren" kurz vor. Hierbei wurde dem Konzept zugrundegelegt, daß mit moderner Technik Temperaturen, die experimentell die Chemotherapiewirkung im Tumor dramatisch verstärken, erreicht werden können. Die brennende Frage der Diskussionsrunde war dabei nach der Höhe der nötigen Temperatur zur wirksamen Effektivität, insbesondere aber auch die Frage der möglichen Nebenwirkungen.

Zur Abrundung des komplexen Themas der kindlichen Rhabdomyosarkome zeigte abschließend A. Sigel (Erlangen) 2 Fallbeispiele zum „Diskussionsdia Makropathologie“.
Dr. D. Sternberg

Urologische Klinik und

Poliklinik der Universität Mainz

Langenbeckstr. 1

D-55131 Mainz 